\title{
Rhinoviruses in infancy and risk of immunoglobulin E sensitization
}

\section{DIABIMMUNE Study Grp}

2019-08

DIABIMMUNE Study Grp , Korhonen , L , Oikarinen , S , Lehtonen , J , Mustonen , N , Tyni , I , Niemelä , O , Honkanen , H , Huhtala , H , llonen , J , Hämäläinen , A-M , Peet , A , Tillmann , V , Siljander , H , Knip , M , Lönnrot , M , Hyöty , H , Härkönen , T , Ryhänen , S , Koski , K, Kiviniemi , M , Ahlfors , H, Kallionpää , H, Laajala, E, Lahesmaa , R , Lähdesmäki , H , Moulder , R, Nieminen , J , Ruohtula , T, Vaarala , O , Alahuhta , K, Virtanen , S M \& Kondrashova , A 2019 , ' Rhinoviruses in infancy and risk of immunoglobulin E sensitization ', Journal of Medical Virology , vol. 91 , no. 8 , pp. 1470-1478 . https://doi.org/10.1002/jmv.25455

http://hdl.handle.net/10138/318042

https://doi.org/10.1002/jmv.25455

acceptedVersion

Downloaded from Helda, University of Helsinki institutional repository.

This is an electronic reprint of the original article.

This reprint may differ from the original in pagination and typographic detail.

Please cite the original version. 
Laura Korhonen ORCID iD: 0000-0002-5566-8054

\section{Rhinoviruses in infancy and risk of IgE sensitization}

Short title: Rhinoviruses in infancy and atopy

Laura Korhonen $^{1,2,3}$, Sami Oikarinen ${ }^{1,4}$, Jussi Lehtonen ${ }^{1}$, Neea Mustonen ${ }^{5,6}$, Iiris Tyni

${ }^{1}$, Onni Niemelä ${ }^{7}$, Hanna Honkanen ${ }^{1}$, Heini Huhtala ${ }^{8}$, Jorma Ilonen ${ }^{9}$, Anu-Maaria

Hämäläinen ${ }^{10}$, Aleksandr Peet ${ }^{11,12}$, Vallo Tillmann ${ }^{11,12}$, Heli Siljander ${ }^{5,6}$, Mikael Knip

5,6,13,14* , Maria Lönnrot ${ }^{1,2,3^{*}}$, Heikki Hyöty ${ }^{1,4^{*}}$ on behalf of the Diabimmune Study

Group $^{\S}$

${ }^{1}$ Department of Virology, Faculty of Medicine and Life Sciences, University of Tampere, Tampere, Finland

${ }^{2}$ Department of Dermatology, Tampere University Hospital, Tampere, Finland

${ }^{3}$ Allergy Centre, Tampere University Hospital, Tampere, Finland

${ }^{4}$ Fimlab Laboratories Ltd, Department of Clinical Microbiology, Tampere, Finland

${ }^{5}$ Children's Hospital, University of Helsinki and Helsinki University Hospital, Helsinki, Finland

${ }^{6}$ Research Programs Unit, Diabetes and Obesity, University of Helsinki, Helsinki, Finland

${ }^{7}$ Department of Laboratory Medicine and Medical Research Unit, Seinäjoki Central Hospital and University of Tampere, Seinäjoki, Finland

${ }^{8}$ Faculty of Social Sciences, University of Tampere, Tampere, Finland

${ }^{9}$ Immunogenetics Laboratory, University of Turku and Turku University Hospital, Turku, Finland

${ }^{10}$ Department of Pediatrics, Jorvi Hospital, Helsinki University Hospital, Espoo, Finland

This article has been accepted for publication and undergone full peer review but has not been through the copyediting, typesetting, pagination and proofreading process, which may lead to differences between this version and the Version of Record. Please cite this article as doi: 10.1002/jmv.25455. 


\author{
${ }^{11}$ Department of Pediatrics, University of Tartu, Tartu, Estonia \\ ${ }^{12}$ Children’s Clinic of Tartu University Hospital, Tartu, Estonia \\ ${ }^{13}$ Folkhälsan Research Center, Helsinki, Finland \\ ${ }^{14}$ Department of Pediatrics, Tampere University Hospital, Tampere, Finland \\ *Should be considered joint senior author \\ ${ }^{\S}$ All authors and their affiliations appear at the end of the article.
}

\title{
Corresponding author:
}

Laura Korhonen, Department of Dermatology, Tampere University Hospital, PO Box 2000, FI-33521, Tampere, Finland. Telephone: +358 3311 66469, fax number: +358 3 3116 5654. Email: laura.korhonen@sll.fimnet.fi

\begin{abstract}
Previous data about the role of viruses in the development of allergic IgE sensitization are contradictory. The aim of this study was to determine the possible associations between exposure to different viruses (rhinovirus, enterovirus, norovirus and parechovirus) during the first year of life and IgE sensitization. Viruses were analyzed from stool samples collected monthly from infants participating in a prospective birth cohort study. From that study, 244 IgE sensitized case children and 244 non-sensitized control children were identified based on their allergen specific IgE antibody levels at the age of 6, 18 and 36 months. Stool samples $(n=4576)$ from case and control children were screened for the presence of rhinovirus, enterovirus, norovirus and parechovirus RNA by RT-qPCR. The study showed that rhinovirus was the most prevalent virus detected, present in 921 (20\%) samples. None of the viruses were associated with IgE sensitization in the full cohort but after stratifying by sex, the number of rhinovirus positive samples
\end{abstract}

This article is protected by copyright. All rights reserved. 
was inversely associated with IgE sensitization in boys [odds ratio (OR): 0.81; 95\% confidence interval (CI): 0.69-0.94; $p=0.006]$. There was also a temporal relation between rhinoviruses and IgE sensitization, as rhinovirus exposure during the first 6 months of life was associated with a reduced risk of subsequent IgE sensitization in boys (OR, 0.76; 95\% CI, 0.6-0.94; $p=0.016$ ). In conclusion, early exposure to rhinoviruses was inversely associated with IgE sensitization but this protective association was restricted to boys.

Keywords: Allergy, atopy, gender, sex, stool, virus

\section{Introduction}

The rapid increase in the prevalence of atopic diseases during the last decades highlights the role of environmental factors in the pathogenesis of these diseases. ${ }^{1}$ IgE sensitization is a marker of atopic constitution and is often associated with atopic disease, such as asthma, allergic rhinoconjuctivitis and atopic eczema. Viral infections are among the potentially interesting environmental exposures affecting IgE sensitization but current data on the associations between viruses and IgE sensitization are conflicting. Some studies have shown virus infections to associate with a lower risk of IgE sensitization, ${ }^{2,3}$ whereas others have observed no associations ${ }^{4,5}$ or an increased risk. ${ }^{6-8}$ There are also indications that viruses may differ in their ability to modulate the risk of IgE sensitization. $^{3,6,7}$

The aim of this study was to evaluate the associations between viral infections in infancy and IgE sensitization. The gut immune system is important in the development of immunological tolerance in early childhood and gastrointestinal viruses have been suggested to be of special significance with regard to atopic sensitization. ${ }^{9-11}$ Therefore, 
viruses known to replicate in the gastrointestinal tract were selected for the present study: Enteroviruses (family Picornaviridae, genus Enterovirus, species Enterovirus A-D), noroviruses (family Caliciviridae, genus Norovirus) and parechoviruses (family Picornaviridae, genus Parechovirus, species Parechovirus A). Rhinoviruses (RVs) belong to the Enterovirus genus of the Picornaviridae family, and they are classified into three species: Rhinovirus A-C. Wheezing RV-infections have been shown to increase the risk of childhood asthma, ${ }^{12,13}$ and they have been reported to be present in fecal samples. ${ }^{14-17}$ Thereby, also RVs were included in the present study, even though there is no evidence that they could replicate in the intestinal mucosa, but are rather passively transmitted from their replication site in the respiratory mucosa into the gastrointestinal tract. In this study, RV, enterovirus (EV), norovirus (NoV) and parechovirus (HPeV) RNA was analyzed by RT-qPCR from stool samples collected monthly during the first year of life from IgE sensitized and non-sensitized children. To our knowledge, there are no previous studies about the associations between viruses detected in stool and risk of IgE sensitization.

\section{Materials and Methods}

\section{Subjects}

The study was carried out as a part of the Diabimmune study aimed at delineating environmental factors predisposing to immune-mediated diseases such as allergies and type 1 diabetes (T1D). ${ }^{18}$ HLA DR-DQ genotypes associated with T1D were screened from the cord blood of 5819 newborns in Finland and Estonia, as described earlier. ${ }^{18}$ All children with designated T1D-related HLA DR-DQ genotypes ( $n=1139)$ were invited to participate in the Diabimmune birth cohort study. Infants were categorized as having 
high (DR3-DQ2/DR4-DQ8 genotype), moderate (DR4-DQ8/X genotype; $\mathrm{X}$ = nonprotective allele but not DR3-DQ2) or slightly increased (DR3-DQ2/Y genotype; $\mathrm{Y}=$ non-protective allele but not DR4-DQ8) risk for T1D. The study was approved by the local Ethics Committees (228/13/03/03/2008 and 172/T-15; 20.08.2008) and the parents gave their written informed consent.

Altogether 717 out of the 1139 HLA-eligible newborns participated in the birth cohort study and 563 children continued until the end of the follow-up at 36 months. For the present study, children were selected according to their IgE levels among these 563 children. In the Diabimmune study, allergen specific IgE levels were measured at 6, 18 and 36 months of age. Altogether 244 (43\%) children had positive IgE values against at least one allergen at the age of 18 and/or 36 months. These 244 children comprised the case group. Next, 244 non-IgE sensitized control children were selected, i.e. control children had negative allergen specific IgE levels at 6, 18 and 36 months. Case and control children were matched for the country of birth, 304 children from Finland and 184 children from Estonia, but otherwise control children were selected randomly. All children were born between September 2008 and May 2010. Demographic characteristics of the case and control groups are presented in Table 1.

\section{Virus analyses}

Stool samples collected during the first 12 months of life were systematically screened for the presence of RV, EV, NoV and HPeV RNA using RT-qPCR. Viral RNA was extracted from 10\% stool suspension in HANK's solution with Qiagen Viral RNA kit (Qiagen, Germany) according to manufacturer’s instructions. Viral RNA was reversetranscribed and amplified according to manufacturer's protocol with QuantiTect Probe 
kit (Qiagen, Germany) using primers and labelled probes (Thermo Fisher Scientific, USA). RV was detected by forward primer 5'-CYA* GCC T*GC GTG GC -3' (A* and T* locked nucleic acid primer by Exiqon); reverse primer GAA ACA CGG ACA CCC AAA GTA and probe VIC-TCC TCC GGC CCC TGA ATG YGG C -TAMRA. ${ }^{19}$ For EV, the primers and probes applied were: forward primer: CGG CCC CTG AAT GCG GCT AA, reverse primer: GAA ACA CGG ACA CCC AAA GTA, probe 1: FAM-TCT GTG GCG GAA CCG ACT A-TAMRA, probe 2: FAM-TCT GCA GCG GAA CCG ACT A-TAMRA. ${ }^{20}$ Primers and probes for NoV and $\mathrm{HPeV}$ detection were applied as described earlier. ${ }^{21}$ All samples were tested in three reactions and if any of the reactions was positive that sample was interpreted as positive for tested virus. The samples were analyzed blind to case-control status of the child.

\section{IgE antibodies}

Serum samples taken at the age of 6, 18 and 36 months were analyzed by ImmunoCAP ${ }^{\circledR}$ enzyme immunoassay (Thermo Fisher Scientific, Uppsala, Sweden) for IgE antibodies against cat, dust mite, birch, timothy, egg, milk and wheat. IgE antibodies against peanut were analyzed at the age of 18 and 36 months and antibodies against dog at 36 months. All analyses were carried out blind to clinical information. Values $\geq 0.35 \mathrm{kU} / \mathrm{L}$ were considered positive.

\section{Statistical methods}

Logistic regression analysis was applied to estimate odds ratios (ORs) and corresponding 95\% confidence intervals (CIs) for IgE sensitization. The association between virus exposure and IgE sensitization was evaluated by calculating the number of virus positive stool samples in each child during the follow-up and comparing the number of positive 
samples in case and control children. After stratification by sex, this comparison was made between male cases and controls and accordingly, between female cases and controls. OR value expresses an estimation of how much each virus positive sample affects the risk to become IgE sensitized. This estimate was calculated for each virus separately. Additionally, the number of case and control children having at least one virus positive sample during the follow-up was calculated for each of the four viruses and logistic regression analysis was applied to estimate the ORs and 95\% CIs for IgE sensitization.

IgE values were measured at 6,18 and 36 months of age and viruses were analyzed from stool samples collected monthly during the first year of life (Figure 1). To observe temporal relations between virus exposure and IgE sensitization, case children were divided into two groups; late sensitized $(n=162)$ and early sensitized ( $n=57)$ (IgE values at 6 months were missing from 25 cases and 10 controls). The late sensitized group comprised of case children who were IgE negative at 6 months but became IgE positive later during the follow-up, and the early sensitized children were case children who were IgE positive already at 6 months. In the late and early sensitized groups, the associations between virus exposure and IgE sensitization were determined by calculating the number of virus positive samples during the first 6 months of follow-up.

Children were also classified according to the nature of their IgE sensitization: children sensitized against at least one aeroallergen (cat, dog, dust mite, birch, timothy) were classified as "aeroallergen sensitized" ( $n=104)$ and children sensitized against at least one dietary allergen (egg, milk, wheat, peanut) comprised the group of “dietary sensitized” ( $n=214)$. Depending on their sensitization profile, case children could belong to one or both of these groups (Supplement Table 1).

This article is protected by copyright. All rights reserved. 
Demographic characteristics of the study population are shown in Table 1 . These data included: T1D-related HLA risk classes, parental atopy (having at least one of the following: asthma, hay fever/allergic rhinitis or atopic dermatitis), having older siblings, smoking inside, place of residence (“urban”: city center or residential areas, “rural” : population centers/sparsely populated areas and "both": change between the categories during the follow-up), type of housing, having furry pets at home, child’s age at the beginning of daycare, duration of breastfeeding (including partial breastfeeding), route of delivery, duration of pregnancy and birth weight. These factors were also addressed as possible confounding factors. Univariate logistic regression analysis was applied to estimate the ORs and 95\% CIs for each factor (Table 1). When a significant effect on IgE sensitization was observed, logistic regression analysis was used to adjust for this factor. Unadjusted $p$ values are presented in the text.

The Bonferroni correction was applied to counteract the problem of multiple comparisons. As the association between virus exposure and IgE sensitization was analyzed for 4 different viruses, the Bonferroni correction to control type I error was justified. After Bonferroni correction, $p<0.013$ was considered statistically significant. Analyses were performed by using R version 3.3.3 (2017-03-06, The R Foundation for Statistical Computing, https://www.R-project.org).

\section{Results}

Altogether 4576 stool samples were analyzed, 2258 from case children and 2318 from control children. The number of stool samples available for analysis each month is presented in Supplement Table 2. RV was the most prevalent virus detected during the first year of life, with 921/4576 (20\%) positive samples. The number of positive samples 
for EV, NoV and HPeV was 203 (4\%), 243 (5\%) and 194 (4\%), respectively. RVs were prominent already during the first 6 months of life, whereas the prevalence of EVs, NoVs and HPeVs started to increase later (Figure 2).

The number of children having at least one virus positive sample during the follow-up did not differ between case and control children for any of the viruses. For RVs: 184 (75\%) case children vs. 187 (77\%) control children had at least one positive sample (OR: 0.93, 95\% CI: 0.62-1.42, $p=0.75$ ), for EVs: 57 (23\%) case children vs. 51 (21\%) control children (OR: 1.15; 95\% CI: 0.75-1.77, $p=0.51$ ), for NoVs: 69 (28\%) case children vs. 83 (34\%) control children (OR: 0.76; 95\% CI: 0.52-1.12, $p=0.17$ ) and for HPeVs: 62 (25\%) case children vs. 66 (27\%) control children (OR: 0.92; 95\% CI: 0.61-1.38, $p=0.68)$.

The number of virus positive stool samples during the follow-up did not differ between case and control children for any of the viruses in the full cohort (Table 2). However, after stratifying the study cohort by sex, the number of RV positive samples was inversely associated with IgE sensitization in boys (OR: 0.81; 95\% CI: 0.69-0.94; $p=0.006$ ) but not in girls (OR: 1.02; 95\% CI: 0.87-1.19; $p=0.83)$. In boys, 104 (74\%) case children had at least one RV positive stool sample vs. 89 (84\%) control children. In girls, 80 (77\%) case children had at least one RV positive samples vs. 98 (71\%) in control children. Sex did not influence the overall prevalence of RV infections, as the number of RV positive samples did not differ between boys and girls: 485 (21\%) vs. 436 (19\%), respectively. Stratification by sex did not have an effect on the associations observed with EV, NoV or HPeV (Table 2). The demographic characteristics stratified by sex are presented in Supplement Tables 3 and 4. The number of case and control children with 
positive paternal history of atopy differed statistically significantly in the full cohort and among girls. Adjusting for this confounder did not affect the results.

The sex-based difference in the association between RV exposure and IgE sensitization was observed also in the late and early sensitized groups (definitions in Supplement Table 1). In boys, the number of RV positive samples during the first 6 months of life was inversely associated with subsequent IgE sensitization (late sensitized group): OR: 0.76; 95\% CI: 0.6-0.94; $p=0.016$, and simultaneous IgE sensitization (early sensitized group): OR: 0.6; 95\% CI: 0.39-0.85; $p=0.008$. In girls, no associations were observed. In addition, an inverse association between RV positivity and IgE sensitization was detected in both aeroallergen and dietary sensitized boys (OR: 0.78; 95\% CI: 0.63-0.94; $p=0.014$ and OR: 0.82; 95\% CI: 0.69-0.95; $p=0.012$, respectively), whereas no association was observed in girls (Figure 3). The number of samples positive for $\mathrm{EV}$, NoV or $\mathrm{HPeV}$ was not associated with IgE sensitization in any of the case categories (data not shown).

\section{Discussion}

The results from this prospective birth cohort study reinforce previous data that RVs are frequent in stool in infancy. The study demonstrates for the first time that RVs detected in stool are inversely associated with IgE sensitization. The study also reports a novel sex-based difference, as the inverse association between RV exposure and IgE sensitization was only observed in boys. Furthermore, RV detection during the first 6 months of life was inversely associated with subsequent IgE sensitization in boys. This temporal order, i.e. RV exposure preceding IgE sensitization, suggests that RV infections in early life may act as protective environmental factors against IgE sensitization, but the effect seems to be associated with sex.

This article is protected by copyright. All rights reserved. 
Previous studies have shown that RVs, although considered respiratory pathogens, are detected frequently in stool in childhood. ${ }^{14-17}$ The reasons for this phenomenon are not clear. RVs infect primarily nasopharyngeal epithelia, and RVs passing through the gastrointestinal tract is the most plausible explanation for detecting RV in stool. RVs are also acid sensitive and thereby thought to degrade in the acidic gastric environment. One explanation for the high detection rate of RVs in stool in early life might lie in the relatively high gastric $\mathrm{pH}$ in infancy that might lead to diminished degradation of $\mathrm{RV}$ RNA in the stomach. ${ }^{22}$ The prospective study setting in the present study allowed also detecting temporal changes in the virus prevalence; the number of RV positive stool samples was high in early infancy and gradually decreased during the first year of life. The observed decreasing RV prevalence with age may well be explained by a steep decrease in gastric $\mathrm{pH}$ during first year of life, observed by Nagita et al. ${ }^{22}$ There is also an in vitro study showing that acidic environment selects rapidly RV strains carrying a point mutation in the capsid protein conferring resistance to inactivation in low $\mathrm{pH} .{ }^{23}$ In addition to acid sensitivity, RVs have been thought to replicate optimally at temperatures of $33-34^{\circ} \mathrm{C}$ equaling that of the upper airways. However, Papadopoulos et al reported only minimal differences in the replication capacity of some RVs between temperatures of $33^{\circ} \mathrm{C}$ and $37^{\circ} \mathrm{C} .{ }^{24}$ Even though not addressed in the current study, it may therefore be that some RV types also retain their viability in the gut, as suggested by the results showing RVs replicating upon cultivation after isolation from stool. ${ }^{15,16}$

$\mathrm{RV}$-induced wheezing has been shown to increase the risk of childhood asthma ${ }^{25}$ but there are much less data about the role of RVs in IgE sensitization. ${ }^{4,7,26}$ Jartti et al reported a positive association between wheezing RV infection and IgE sensitization in a cross-sectional study setting. ${ }^{7}$ Later, a prospective study showed that aeroallergen 
sensitization predisposed children to RV-induced wheezing, but the opposite was not seen. ${ }^{4}$ It might also be important to notice, that most previous studies have relied on samples taken during symptomatic RV infections, especially RV-induced wheezing, with less data derived from systematic sample collection regardless of symptoms. ${ }^{13,27}$ In fact, there are indications that asymptomatic or mild RV infections might differ from severe ones in relation to atopy. Jackson et al reported severe wheezing RV infections to increase the risk of asthma but found no association between asthma and RVs detected from nasal samples during prescheduled visits. ${ }^{13}$ In accordance, picornaviruses, including RVs, detected from routine nasal samples taken at four distinct time points during the first year of life, were shown not to associate with atopic sensitization. ${ }^{27}$ Thus, it is possible that the association between RVs and wheezing episodes is characteristic for children who already have airway hyper reactivity, while RV infections that occur in early infancy may reduce the risk of IgE sensitization. The latter association may be more difficult to detect since it requires prospective birth cohort studies and depends on the sex of the child.

The sex-based difference observed is interesting, since susceptibility to a wide range of infections and immune-mediated diseases is influenced by sex. In general, boys have increased susceptibility to many virus infections whereas girls often develop stronger immune responses resulting in lower prevalence and intensity of infections. ${ }^{28,29}$ There are not much prior data about possible sex-based differences in RV infections. Boys were shown to have more moderate-to-severe RV illnesses during early childhood as well as higher IFN- $\gamma$ responses than girls did. ${ }^{30}$ In addition, an animal study showed that early RV infections contributed to the development of allergen induced lung disease in female, but not in male mice. ${ }^{31}$ On the other hand, there is a well-known sex-based difference in 
the prevalence of IgE sensitization and childhood asthma with male gender predisposing to both. ${ }^{30,32,33}$ The reasons for the male dominance in susceptibility to virus infections and atopy are not known but diverse and widespread effects of sex hormones probably play a role. ${ }^{34}$

Most previous birth cohorts on atopy have included children genetically at high risk for atopy. There is a strong and heterogeneous genetic component in childhood asthma ${ }^{35}$ and maternal atopy has also been reported to increase the risk of severe RV bronchiolitis. ${ }^{36}$ Furthermore, variants at the $17 q 21$ gene locus have been shown to associate with asthma especially in children presenting with RV-associated wheezing illness. ${ }^{37}$ It is therefore possible that in genetically selected cohorts, children are not only at higher risk for asthma but also for severe RV infections, which could contribute to the previously observed positive associations between RV infections and asthma. On the other hand, the study population in the present study was selected for T1D-associated HLA types. There are some data linking HLA-DQ region to asthma, and HLA-DRB1 has also been linked to IgE sensitization. ${ }^{38}$ However, as the genetic background of atopy is highly polymorphic and it has been estimated that HLA-DRB1 locus only accounts for 2-3\% of the variation in specific IgE titers, ${ }^{38}$ it is unlikely that the HLA selection would have substantially influenced our results. Furthermore, the distribution of HLA-DR/DQ types was similar in case and control groups.

RV infections are generally diagnosed by RT-PCR assays targeting the 5' non-coding region (5'-NCR) of the RV genome. ${ }^{39}$ Due to sequence similarities in the 5'NCR between RVs and EVs, it is known that some RV PCR assays detect also EVs thereby reducing the specificity of the assays. In a study by Lu et al, the RV RT-PCR assay applied in the present study was shown to amplify all of the $100 \mathrm{RV}$ prototype strains and 
85 field isolate strains included in their sensitivity and specificity analysis. ${ }^{19}$ The assay did not amplify 34 of the 48 EV types included, while 14 EV types gave weakly positive reactions when present in the sample in high titres. ${ }^{19}$ In addition, the RV RT-PCR primers and probes applied in the present study were included in the study by Faux et al comparing the usefulness of different PCR primers in detecting RV infections. ${ }^{40}$ The RV RT-PCR assay in question, amplified 20 out of the 29 RV types including types from all RV species (Rhinovirus A-C) and most importantly, the assay did not amplify any of the EVs included. ${ }^{40}$ These studies confirm that the RV RT-PCR primers and probes applied in the current study are both sensitive and specific. However, the possibility that some RVs might have been missed or that there might be some low-degree cross-detection between RVs and EVs cannot be excluded.

In the current study, no genotyping of RVs was performed. Thereby, the results do not indicate whether consecutive RV positive stool samples represent a single prolonged infection or separate independent infections. However, in a former study by our group, 43 different RV types were identified among the $63 \mathrm{RV}$ positive stool samples sequenced and only once was the same genotype detected in two sequential samples. ${ }^{15}$ This finding by Honkanen et al is an illustration of the high genetic diversity characteristic for RVs and suggests that shedding of RVs in stool is short-term. These previous results support the current interpretation that each RV positive sample represents individual exposure and the high frequency of RVs in stool probably reflects the diversity of circulating RVs.

The allergen panel applied in the IgE analysis was defined according to local sensitization patterns against dietary and aeroallergens. ${ }^{41}$ It includes majority of the relevant allergens, and is thereby likely to detect most IgE sensitized children in this age

This article is protected by copyright. All rights reserved. 
group. However, some children sensitized only to some of the more uncommon allergens might have been missed but the number of these children is estimated to be low.

In conclusion, this study provides new insights into the role of different viruses, especially RVs, in atopic sensitization. Data based on monthly collection of stool samples demonstrated that RVs are commonly present in young infants' gut and that this $\mathrm{RV}$ exposure was associated with a reduced risk of IgE sensitization in boys. The findings suggest that RVs might have a more versatile role in the development of atopy than previously perceived. We hypothesize that early RV exposure could serve a beneficial function in driving postnatal maturation of the immune competence, thereby reducing the risk of allergic sensitization. In addition, the clear sex-based difference in the effect of RVs observed emphasizes the importance of exploring the impact of sex also in future studies aimed at elucidating the mechanisms of atopic disease.

\section{Funding}

This work was funded by the European Union Seventh Framework Program FP7/20072013 [grant agreement no. 202063], Tampere Tuberculosis Foundation, Sigrid Juselius Foundation, Academy of Finland [Prevaller consortium, grant 129448-2008; Research grant 132362-2009; Centre of Excellence in Molecular Systems Immunology and Physiology, grant 250114 2012-2017], The National Technology Agency in Finland (TEKES), Päivikki and Sakari Sohlberg Foundation, Orion-Farmos Research Foundation and the Estonia Research Council grant PUT 1382.

\section{Conflict of interest}

Professors Hyöty and Knip are minor shareholders of Vactech Ltd developing vaccines against picornaviruses. Other authors declare none.

This article is protected by copyright. All rights reserved. 


\section{Acknowledgements}

The authors would like to thank the DIABIMMUNE study group. The authors are grateful to Katriina Koski and Matti Koski for their valuable assistance in database work and to Pekka Keränen, Tanja Kuusela and Maria Ovaskainen for their skillful technical assistance. The authors would like to thank also the local study personnel and the participating families for their collaboration.

\section{Diabimmune Study Group}

Mikael Knip, Taina Härkönen, Samppa Ryhänen and Heli Siljander (Children’s Hospital, University of Helsinki, Helsinki, Finland)

Katriina Koski and Matti Koski (Institute of Clinical Medicine, University of Helsinki, Helsinki, Finland)

Anu-Maaria Hämäläinen (Jorvi Hospital, Helsinki University Central Hospital, Espoo, Finland)

Anne Ormisson (Children’s Clinic, Tartu University Hospital, Tartu, Finland)

Aleksandr Peet and Vallo Tillmann (Department of Pediatrics, Tartu University Hospital, Tartu, Finland)

Valentina Ulich, Elena Kuzmicheva and Sergei Mokurov (Ministry of Health and Social Development, Karelian Republic of the Russian Federation, Petrozavodsk, Russia)

Svetlana Markova and Svetlana Pylova (Children’s Republic Hospital, Karelian Republic of the Russian Federation, Petrozavodsk, Russia)

This article is protected by copyright. All rights reserved. 
Marina Isakova and Elena Shakurova (Perinatal Center, Karelian Republic of the Russian Federation, Petrozavodsk, Russia)

Vladimir Petrov (Maternity Hospital No. 1, Petrozavodsk, Russia)

Natalya V. Dorshakova, Tatyana Karapetyan and Tatyana Varlamova (Petrozavodsk State University, Petrozavodsk, Russia)

Jorma Ilonen and Minna Kiviniemi (Immunogenetics Laboratory, University of Turku, Turku, Finland)

Jorma Ilonen (Department of Clinical Microbiology, University of Eastern Finland, Kuopio, Finland)

Kristi Alnek, Helis Janson and Raivo Uibo (Department of Immunology, University of Tartu, Tartu, Estonia)

Tiit Salum (OÜ Immunotron, Tartu, Estonia)

Erika von Mutius and Juliane Weber (Children’s Hospital, Ludwig Maximilians University, Munich, Germany)

Helena Ahlfors, Henna Kallionpää, Essi Laajala, Riitta Lahesmaa, Harri Lähdesmäki and Robert Moulder (Turku Centre of Biotechnology, University of Turku and Åbo Akademi University, Turku, Finland)

Janne Nieminen, Terhi Ruohtula and Outi Vaarala (Department of Vaccination and Immune Protection, National Institute for Health and Welfare, Helsinki, Finland)

This article is protected by copyright. All rights reserved. 
Heikki Hyöty, Hanna Honkanen, Anita Kondrashova and Sami Oikarinen (Department of Virology, University of Tampere, Tampere, Finland)

Hermie J. M. Harmsen, Marcus C. De Goffau and Gjal Welling (University Medical Center Groningen, Groningen, the Netherlands)

Kirsi Alahuhta and Suvi M. Virtanen (Department for Welfare and Health Promotion, National Institute for Health and Welfare, 00271 Helsinki, Finland)

\section{References}

1. Liu AH. Revisiting the hygiene hypothesis for allergy and asthma. J Allergy Clin Immunol. 2015;136:860-865.

2. Nordstrom I, Rudin A, Adlerberth I, et al. Infection of infants with human herpesvirus type 6 may be associated with reduced allergic sensitization and T-helper type 2 development. Clin Exp Allergy. 2010;40:882-890.

3. Nilsson C, Linde A, Montgomery SM, et al. Does early EBV infection protect against IgE sensitization? J Allergy Clin Immunol. 2005;116:438-444.

4. Jackson DJ, Evans MD, Gangnon RE, et al. Evidence for a causal relationship between allergic sensitization and rhinovirus wheezing in early life. Am J Respir Crit Care Med. 2012;185:281-285.

5. Alcantara-Neves NM, Veiga RV, Dattoli VCC, et al. The effect of single and multiple infections on atopy and wheezing in children. J Allergy Clin Immunol. 2012;129:359367.

6. Sidorchuk A, Wickman M, Pershagen G, Lagarde F, Linde A. Cytomegalovirus infection and development of allergic diseases in early childhood: interaction with EBV infection? J Allergy Clin Immunol. 2004;114:1434-1440.

7. Jartti T, Kuusipalo H, Vuorinen T, et al. Allergic sensitization is associated with rhinovirus-, but not other virus-, induced wheezing in children. Pediatr Allergy Immunol. 2010;21:1008-1014.

8. Sigurs N, Gustafsson PM, Bjarnason R, et al. Severe respiratory syncytial virus bronchiolitis in infancy and asthma and allergy at age 13. Am J Respir Crit Care Med. 2005;171:137-141.

This article is protected by copyright. All rights reserved. 
9. Korhonen L, Kondrashova A, Tauriainen S, et al. Enterovirus infections in early childhood and the risk of atopic disease--a nested case-control study. Clin Exp Allergy. 2013;43:625-632.

10. Matricardi PM, Rosmini F, Riondino S, et al. Exposure to foodborne and orofecal microbes versus airborne viruses in relation to atopy and allergic asthma: epidemiological study. BMJ. 2000;320:412-417.

11. Seiskari T, Kondrashova A, Tauriainen S, et al. Role of enterovirus infections in IgE sensitization. J Med Virol. 2012;84:268-271.

12. Kotaniemi-Syrjanen A, Vainionpaa R, Reijonen TM, Waris M, Korhonen K, Korppi M. Rhinovirus-induced wheezing in infancy--the first sign of childhood asthma? $J$ Allergy Clin Immunol. 2003;111:66-71.

13. Jackson DJ, Gangnon RE, Evans MD, et al. Wheezing rhinovirus illnesses in early life predict asthma development in high-risk children. Am J Respir Crit Care Med. 2008;178:667-672.

14. Harvala H, McIntyre CL, McLeish NJ, et al. High detection frequency and viral loads of human rhinovirus species A to $\mathrm{C}$ in fecal samples; diagnostic and clinical implications. J Med Virol. 2012;84:536-542.

15. Honkanen $H$, Oikarinen S, Peltonen $P$, et al. Human rhinoviruses including group $C$ are common in stool samples of young Finnish children. J Clin Virol. 2013;56:334-338.

16. Savolainen-Kopra C, Simonen-Tikka M, Klemola P, et al. Human rhinoviruses in INDIS-study material-evidence for recovery of viable rhinovirus from fecal specimens. J Med Virol. 2013;85:1466-72.

17. Lau SKP, Yip CCY, Lung DC, et al. Detection of human rhinovirus C in fecal samples of children with gastroenteritis. J Clin Virol. 2012;53:290-296.

18. Peet A, Kool P, Ilonen J, Knip M, Tillmann V, for the DIABIMMUNE Study Group. Birth weight in newborn infants with different diabetes-associated HLA genotypes in three neighbouring countries: Finland, Estonia and Russian Karelia. Diabetes Metab Res. 2012;28:455-461.

19. Lu X, Holloway B, Dare RK, et al. Real-time reverse transcription-PCR assay for comprehensive detection of human rhinoviruses. J Clin Microbiol. 2008;46:533-539.

20. Honkanen H, Oikarinen S, Pakkanen O, et al. Human enterovirus 71 strains in the background population and in hospital patients in Finland. J Clin Virol. 2013;56:348353.

21. Krogvold L, Edwin B, Buanes T, et al. Detection of a Low-Grade Enteroviral Infection in the Islets of Langerhans of Living Patients Newly Diagnosed With Type 1 Diabetes. Diabetes. 2015;64:1682-1687.

This article is protected by copyright. All rights reserved. 
22. Nagita A, Amemoto K, Yoden A, et al. Diurnal Variation in Intragastric pH in Children with and without Peptic Ulcers. Pediatr Res. 1996;40:528-532.

23. Skern T, Torgersen H, Auer H, Kuechler E, Blaas D. Human rhinovirus mutants resistant to low pH. Virology. 1991;183:757-763.

24. Papadopoulos NG, Sanderson G, Hunter J, Johnston SL. Rhinoviruses replicate effectively at lower airway temperatures. J Med Virol. 1999;58:100-104.

25. Liu L, Pan Y, Zhu Y, et al. Association between rhinovirus wheezing illness and the development of childhood asthma: a meta-analysis. BMJ Open. 2017;7:e013034.

26. Lee ZM, Huang YH, Ho SC, Kuo HC. Correlation of symptomatic enterovirus infection and later risk of allergic diseases via a population-based cohort study. Medicine (Baltimore). 2017;96:e5827.

27. Lee KK, Hegele RG, Manfreda J, et al. Relationship of early childhood viral exposures to respiratory symptoms, onset of possible asthma and atopy in high risk children: the Canadian Asthma Primary Prevention Study. Pediatr Pulmonol. 2007;42:290-297.

28. Fish EN. The X-files in immunity: sex-based differences predispose immune responses. Nat Rev Immunol. 2008;8:737-744.

29. Muenchhoff M, Goulder PJ. Sex differences in pediatric infectious diseases. $J$ Infect Dis. 2014;209 Suppl 3:S120-126.

30. Uekert SJ, Akan G, Evans MD, et al. RF. Sex-related differences in immune development and the expression of atopy in early childhood. J Allergy Clin Immunol. 2006;118:1375-1381.

31. Phan JA, Kicic A, Berry LJ, Sly PD, Larcombe AN. Early life rhinovirus infection exacerbates house-dust-mite induced lung disease more severely in female mice. Exp Lung Res. 2016;42:24-36.

32. Ronmark E, Warm K, Bjerg A, Backman H, Hedman L, Lundback B. High incidence and persistence of airborne allergen sensitization up to age 19 years. Allergy. 2017;72:723-730.

33. Gabet S, Just J, Couderc R, Seta N, Momas I. Allergic sensitisation in early childhood: Patterns and related factors in PARIS birth cohort. Int J Hyg Environ Health. 2016;219:792-800.

34. Fuseini H, Newcomb DC. Mechanisms Driving Gender Differences in Asthma. Curr Allergy Asthma Rep. 2017;17:19.

35. Moffatt MF, Gut IG, Demenais F, et al. A Large-Scale, Consortium-Based Genomewide Association Study of Asthma. N Engl J Med. 2010;363:1211-1221.

This article is protected by copyright. All rights reserved. 
36. Miller EK, Williams JV, Gebretsadik T, et al. Host and viral factors associated with severity of human rhinovirus-associated infant respiratory tract illness. J Allergy Clin Immunol. 2011;127:883-891.

37. Çalışkan M, Bochkov YA, Kreiner-Møller E, et al. Rhinovirus Wheezing Illness and Genetic Risk of Childhood-Onset Asthma. N Engl J Med. 2013;368:1398-1407.

38. Moffatt MF, Schou C, Faux JA, et al. Association between quantitative traits underlying asthma and the HLA-DRB1 locus in a family-based population sample. Eur $J$ Hum Genet. 2001;9:341-346.

39. Ruuskanen O, Waris M, Ramilo O. New aspects on human rhinovirus infections. Pediatr Infect Dis J. 2013;32:553-5.

40. Faux CE, Arden KE, Lambert SB, et al. Usefulness of Published PCR Primers In Detecting Human Rhinovirus Infection. Emerg Infect Diseases. 2011;17:296-298.

41. Eigenmann PA, Atanaskovic-Markovic M, Hourihane JO'B, et al. Testing children for allergies: why, how, who and when. Pediatr Allergy Immunol. 2013;24:195-209.

\section{Figures}

Figure 1 Time line of the study. Allergen specific IgE levels were measured at 6, 18 and 36 months (mo). Stool samples were collected monthly during the first year of follow-up.

Definition of an atopic case child was IgE sensitization at 18 and/or 36 mo. These case children $(n=244)$ were divided into early sensitized and late sensitized according to their IgE levels at 6 mo (data was missing from 25 case children). Control children were IgE negative at 6, 18 and 36 mo (data at $6 \mathrm{mo}$ was missing from 10 control children).

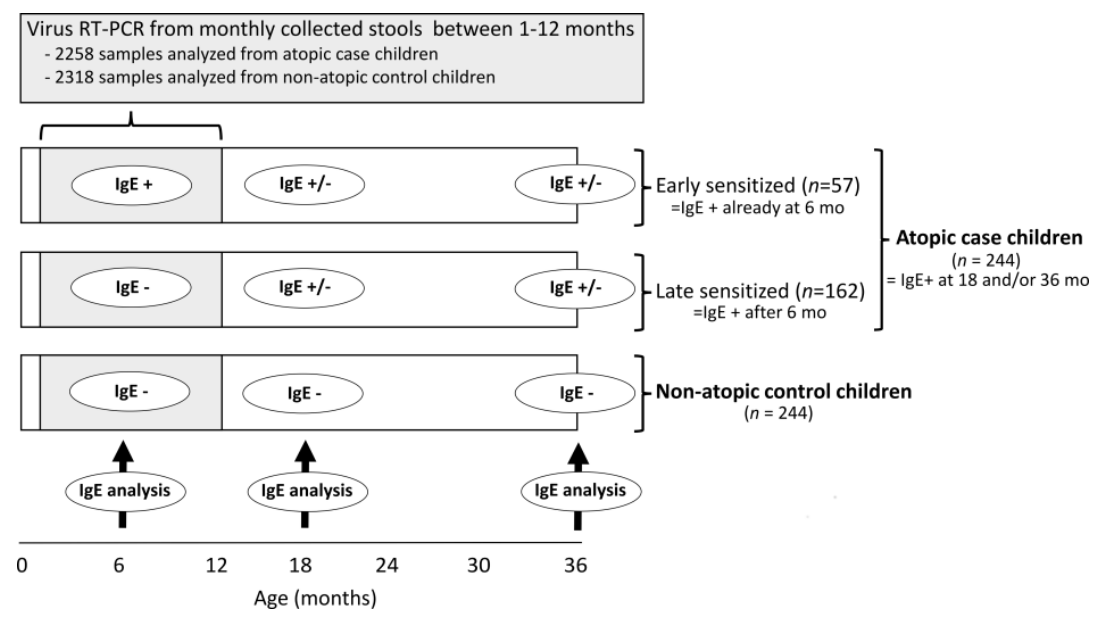

This article is protected by copyright. All rights reserved. 
Figure 2 The percentage of RT-qPCR positive stool samples for each virus during the 12month follow-up. The number of stool samples available for analysis each month is presented in Supplement Table 2 .

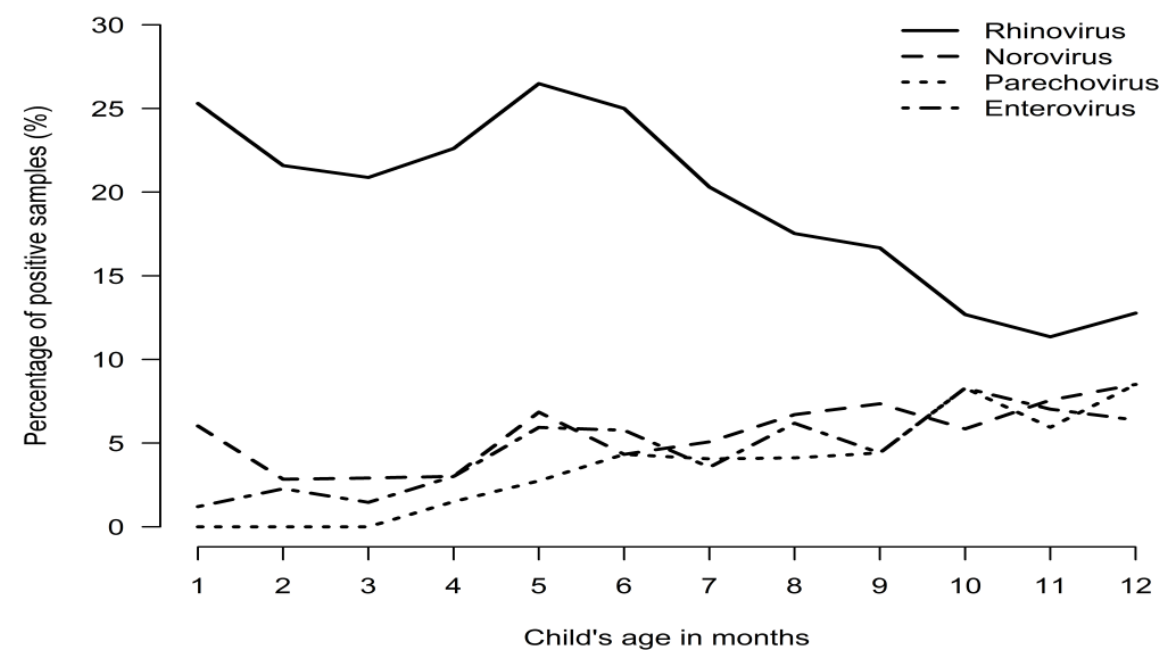

Figure 3 Associations between rhinovirus positive stool samples and IgE sensitization in the full cohort and among boys and girls. Results are expressed as odds ratios (ORs) with 95\% confidence intervals (CIs) for IgE sensitization. Abbreviations: All=all case children, i.e. children IgE sensitized at 18 and/or 36 months (mo), ( $\mathrm{n}=244,140$ boys and 104 girls), early=case children sensitized already 6 months, late=case children sensitized at 18 and/or 36 months but not at 6 months, aero=case children sensitized against aeroallergens, diet $=$ case children sensitized against dietary allergens.

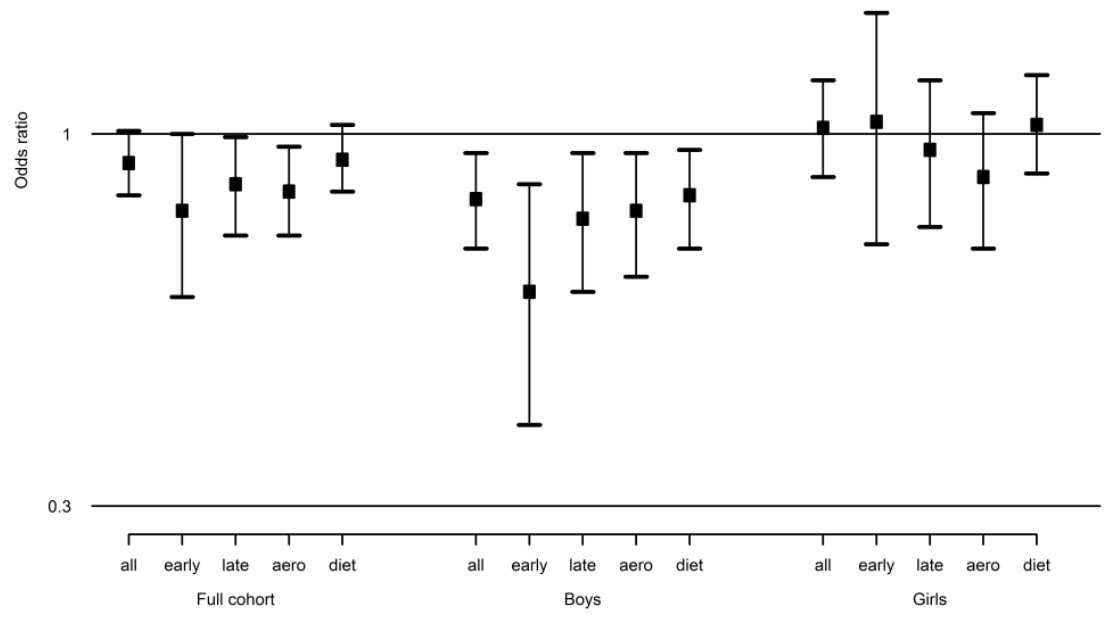

This article is protected by copyright. All rights reserved. 
Table 1.

Table Heading: Demographic characteristics of the study population. The association between each factor and IgE sensitization was estimated by logistic regression analysis (univariate analysis). The results are presented as odds ratio (OR) and 95\% confidence interval (CI) for IgE sensitization.

\begin{tabular}{|c|c|c|c|}
\hline & $\begin{array}{c}\text { Case } \\
n=244(\%)\end{array}$ & $\begin{array}{l}\text { Control } \\
n=244(\%)\end{array}$ & OR $(95 \% \mathrm{CI})$ \\
\hline Gender (male) & $140(57)$ & $106(43)$ & $1.75(1.22-2.51)$ \\
\hline \multicolumn{4}{|l|}{ HLA risk class } \\
\hline DR3-DQ2/DR4-DQ8 & $25(10)$ & $20(8)$ & ref \\
\hline DR4-DQ8/X** & $98(40)$ & $110(45)$ & $0.71(0.37-1.36)$ \\
\hline DR3-DQ2/y** & $121(50)$ & $114(47)$ & $0.85(0.45-1.61)$ \\
\hline Maternal atopy (yes) & $72(30)$ & $72(30)$ & $1(0.68-1.48)$ \\
\hline Paternal atopy (yes) & $77(32)$ & $57(23)$ & $1.53(1.02-2.29)$ \\
\hline \multicolumn{4}{|l|}{ Parental atopy } \\
\hline Neither & $125(52)$ & $136(56)$ & $r e f$ \\
\hline Either or both & $117(48)$ & $108(44)$ & $1.18(0.82-1.68)$ \\
\hline Older siblings (yes) & $156(64)$ & $168(69)$ & $0.8(0.55-1.17)$ \\
\hline Smoking inside (yes) & $16(7)$ & $11(5)$ & $1.51(0.69-3.34)$ \\
\hline \multicolumn{4}{|l|}{ Place of Residence } \\
\hline Urban & $188(79)$ & $194(81)$ & ref \\
\hline Rural & $41(17)$ & $39(16)$ & $1.08(0.67-1.76)$ \\
\hline
\end{tabular}

This article is protected by copyright. All rights reserved. 
Both

Type of Housing

\begin{tabular}{lccc} 
Apartment & $82(34)$ & $93(38)$ & ref \\
Farm & $16(7)$ & $8(3)$ & $2.27(0.92-5.57)$ \\
House or row house & $118(49)$ & $127(53)$ & $1.05(0.71-1.55)$ \\
More than one type & $25(10)$ & $14(6)$ & $2.03(0.99-4.15)$ \\
Pets at home (yes) & $106(44)$ & $98(41)$ & $1.14(0.79-1.63)$ \\
Entry in daycare ${ }^{\S a}$ & $612(463-799)$ & $601(441-836)$ & 1 \\
Cessation of breastfeeding ${ }^{\S a}$ & $313(154-392)$ & $310(148-382)$ & 1 \\
Vaginal delivery (yes) & $224(92)$ & $222(91)$ & $0.9(0.48-1.7)$ \\
Duration of pregnancy ${ }^{\S \#}$ & $281(274-286)$ & $282(274-287)$ & $0.99(0.97-1)$ \\
Birth weight ${ }^{\S}$ q & $3610(3228-3910)$ & $3570(3295-3884)$ & 1 \\
\hline
\end{tabular}

Abbreviations: Case: A child with at least one positive allergen specific IgE level at 18 and/or 36 months, Control: A child with negative allergen specific IgE levels at 6, 18 and 36 months, $\mathrm{x}^{* *}$ not DR3-DQ2 or a haplotype associated with protection against type 1 diabetes (T1D), $\mathrm{y}^{* *}$ not DR4-DQ8 or a haplotype associated with protection against T1D, $\S$ values are medians (interquartile range), a age of the child in days, \# presented in days, 9 presented in grams.

This article is protected by copyright. All rights reserved. 
Table 2.

Table Heading: The number of virus positive samples and associations with IgE sensitization. Each virus was analyzed from stool samples collected during the first year of life ( $n=4576)$. The association between the number of virus positive samples and IgE sensitization is presented as odds ratio (OR) and 95\% confidence interval (CI).

\begin{tabular}{|c|c|c|c|c|}
\hline & Case & Control & & \\
\hline & $n(\%)$ & $n(\%)$ & OR (95\% CI) & $p$ value \\
\hline \multicolumn{5}{|l|}{ Rhinovirus } \\
\hline Full cohort & 429 (19) & 492 (21) & $0.91(0.82-1.01)$ & 0.09 \\
\hline Boys & 239 (19) & $246(24)$ & $0.81(0.69-0.94)$ & 0.006 \\
\hline Girls & 190 (19) & 246 (19) & $1.02(0.87-1.19)$ & 0.83 \\
\hline \multicolumn{5}{|l|}{ Enterovirus } \\
\hline Full cohort & $103(5)$ & $100(4)$ & $1.01(0.80-1.20)$ & 0.89 \\
\hline Boys & $52(4)$ & $42(4)$ & $0.97(0.70-1.30)$ & 0.83 \\
\hline Girls & $51(5)$ & $58(5)$ & $1.07(0.80-1.40)$ & 0.60 \\
\hline \multicolumn{5}{|l|}{ Norovirus } \\
\hline Full cohort & $111(5)$ & $132(6)$ & $0.90(0.70-1.10)$ & 0.29 \\
\hline Boys & $58(5)$ & $59(6)$ & $0.80(0.60-1.10)$ & 0.19 \\
\hline
\end{tabular}

This article is protected by copyright. All rights reserved. 


\begin{tabular}{|c|c|c|c|c|}
\hline Girls & $53(5)$ & $73(6)$ & $1.00(0.70-1.30)$ & 0.88 \\
\hline \multicolumn{5}{|c|}{ Parechovirus } \\
\hline Full cohort & $92(4)$ & $102(4)$ & $0.90(0.70-1.20)$ & 0.55 \\
\hline Boys & $56(4)$ & $51(5)$ & $0.90(0.60-1.21)$ & 0.43 \\
\hline Girls & $36(4)$ & $51(4)$ & $1.00(0.70-1.37)$ & 0.80 \\
\hline
\end{tabular}

This article is protected by copyright. All rights reserved. 